\title{
How can we reduce injuries and illnesses among athletes during the PyeongChang 2018 Olympic Winter Games?
}

\author{
Sun Huh https://orcid.org/0000-0002-8559-8640
}

Korea will host the PyeongChang 2018 Olympic Winter Games from February 9 to February 25, 2018, in which approximately 3,000 athletes are expected to participate. As a medical doctor, I am concerned with the health of all participants. All athletes, who are delegates of their countries, will strive to their utmost potential to perform at the peak of their abilities. In previous Olympics, during the short period of training and competition, the incidence of injuries and illnesses has been very high. Of the 2,567 athletes in the Vancouver 2010 Olympic Winter Games, 287 (11.2\%) were injured and $185(7.2 \%)$ became ill (Engebretsen et al., 2010), while of the 2,788 athletes in the Sochi 2014 Olympic Winter Games, 391 (14.0\%) were injured and 249 (8.8\%) suffered from illness (Fig. 1, Supplementary material) (Soligard et al., 2015). The above data have been made available since 2008 through the injury surveillance system based on the Daily Report on Injuries and Illnesses, the form for which is available at https://stillmed. olympic.org/Documents/Commissions_PDFfiles/Medical_commission/Daily_report_on_Injuries_and_Illnesses-London_2012. pdf (Junge et al., 2008). The daily report form comprises 8 items that should be filled out by a physician or physical therapist as follows: the athlete's accreditation number; sport and event; round, heat, or training; date and time of injury; injured body part (code); type of injury (code); cause of injury (code); and absence in days.

In Vancouver 2010, athletes participating in bobsleigh, ice hockey, short track, alpine freestyle, and snowboard cross were injured more often than those who competed in other events. The most common injury sites were the head, cervical spine, and knee. The most frequent illnesses were respiratory diseases (Engebretsen et al., 2010). In Sochi 2014, athletes competing in aerial skiing, snowboard slopestyle, snowboard cross, slopestyle skiing, halfpipe skiing, moguls skiing, alpine skiing, and snowboard halfpipe were more frequently injured than others. Infection was the most common cause of illness (Soligard et al., 2015). In both Olympic Winter Games, females were more vulnerable to injuries and illnesses (Fig. 1).

I am not sure whether the incidence of injuries and illnesses will increase in PyeongChang 2018. Unfortunately, an athlete passed away during the training period for luge in Vancouver 2010 by bouncing off of the course. In PyeongChang 2018, no such accidents will occur due to dangerous structures. How can we prevent injuries and illnesses among athletes during PyeongChang 2018? This is a difficult question, because they can be expected to strive for the best possible performance without considering their health or safety. For injury prevention, it is known that guidelines for proper training, performance, and rehabilitation should be taught and followed (Jee, 2016). In addition, I suggest the following measures for ensuring the health and safety of athletes in the Olympic Winter Games. First, all athletic structures should be re-assessed in terms of whether they contain dangers or pose certain risks. For example, the forward speed in sled and alpine ski should be mitigated. Second, rules of ice hockey allowing bodychecking should be limited. Third, during short track, athletes should be trained to avoid any dangerous behavior; furthermore, penalties for dangerous playing should be enforced. Due to the high speed of athletes participating in short track, falling onto the ice may cause serious injuries. Success in most games in the Olympic Winter Games is dependent on personal performance, with a few exceptions. Ice hockey allows contact between players, 


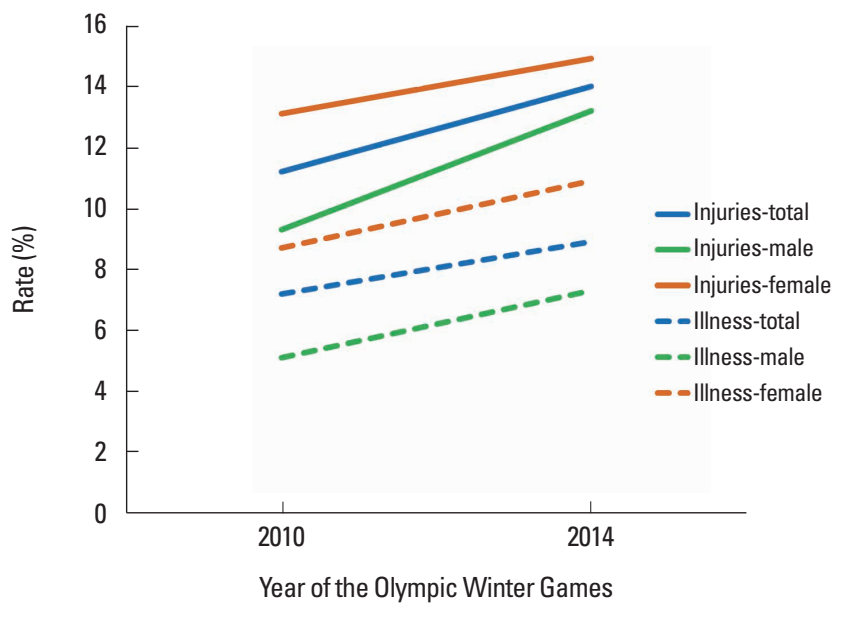

Fig. 1. Incidence of injuries and illnesses during the Olympic Winter Games based on the daily report system in 2010 and 2014, according to the data of Engebretsen et al. (2010) and Soligard et al. (2015).

and it is possible for players to come into contact in short track. Therefore, the rules of those two events should be carefully enforced to provide a safer environment.

The most common cause of illness was respiratory diseases in Vancouver 2010 and infections in Sochi 2014. I believe that those two disease entities simply reflect a difference in classification. To avoid diseases of the upper respiratory system such as the common cold, laryngitis, rhinitis, or pneumonia, it is recommended that all athletes, staff members, and volunteers adhere to the following principles: first, always wear a mask except for during training and competition; second, do not shake hands without a glove because hands can be sources of infection; and third, wash hands frequently with soap. If all participants follow these guidelines, there may be a dramatic decrease in upper respiratory disease incidence.

Not only the athletes but also the audience, enjoy an acrobatic, speedy, and dynamic style of play. The Winter Olympic Games have become more exciting and stimulating. They may now receive more interest from people throughout the world; however, the health and safety of athletes should be considered as the top priority by the International Olympic Committee (IOC).

Exercise rehabilitation plays an important role in helping athletes recover from their injuries. Before rehabilitation, preventing injuries is the best measure to be taken to promote athletes' health and safety. I welcome athletes and supporting staff members visit- ing PyeongChang 2018 from all over the world, and I hope that they enjoy the Olympic Winter Games and their stay in Korea, and that they return home happy and in good health, free from injuries and illness. I also anticipate that the final analysis of injuries and illnesses among athletes who compete at PyeongChang 2018 by the Medical and Scientific Commission of the IOC will show a lower incidence rate than was observed for Vancouver 2010 and Sochi 2014.

\section{CONFLICT OF INTEREST}

No potential conflict of interest relevant to this article was reported.

\section{SUPPLEMENTARY MATERIAL}

Supplementary material can be found via https://oi.org/10.12965/ jer.1735184.592. Total number of athletes in the Olympic Winter Games, Vancouver 2010 and Sochi 2014, and their injuries and illnesses according to gender.

\section{REFERENCES}

Engebretsen L, Steffen K, Alonso JM, Aubry M, Dvorak J, Junge A, Meeuwisse W, Mountjoy M, Renström P, Wilkinson M. Sports injuries and illnesses during the Winter Olympic Games 2010. Br J Sports Med 2010;44:772-780.

Jee H. The science behind the Olympic glory. J Exerc Rehabil 2016;12:253254.

Junge A, Engebretsen L, Alonso JM, Renström P, Mountjoy M, Aubry M, Dvorak J. Injury surveillance in multi-sport events: the International Olympic Committee approach. Br J Sports Med 2008;42:413-421.

Soligard T, Steffen K, Palmer-Green D, Aubry M, Grant ME, Meeuwisse W, Mountjoy M, Budgett R, Engebretsen L. Sports injuries and illnesses in the Sochi 2014 Olympic Winter Games. Br J Sports Med 2015;49:441447.

Department of Parasitology and Institute of Medical Education, Hallym University College of Medicine, 1 Hallimdaehak-gil, Chuncheon 24252, Korea E-mail: shuh@hallym.ac.kr 